\title{
Could Pentraxin 3 Be a New Diagnostic Marker for Excessive Inflammatory Response in Cardiac Surgery?
} \author{
Tomas Holubec ${ }^{3}$ \\ ${ }^{1}$ Department of Cardiac Surgery, Charles University in Prague, Faculty \\ of Medicine and University Hospital Hradec Kralove, Hradec Kralove, \\ Czech Republic \\ 2 Department of Clinical Immunology and Alergology, Charles \\ University in Prague, Faculty of Medicine and University Hospital \\ Hradec Kralove, Hradec Kralove, Czech Republic \\ ${ }^{3}$ Division of Cardiovascular Surgery, University Hospital Zurich, \\ Zurich, Switzerland \\ Thorac Cardiovasc Surg 2014;62:670-676.
}

Zdenka Holubcova ${ }^{1}$ Pavel Kunes ${ }^{1}$ jiri Mandak ${ }^{1}$ Martina Kolackova ${ }^{2}$ Ctirad Andrys $^{2}$ Jan Krejsek ${ }^{2}$

\begin{abstract}
Address for correspondence Tomas Holubec, MD, PhD, Division of Cardiovascular Surgery, University Hospital Zurich, Raemistrasse 100, Zurich 8091, Switzerland (e-mail: tomasholubec@email.cz).
\end{abstract}

\begin{abstract}
Keywords

- SIRS

- postoperative care and complications

- cardiac surgery

Background The aim of this study was to compare the dynamics of two inflammatory response biomarkers pentraxin 3 (PTX3) and C-reactive protein (CRP) after cardiac surgery with particular regard to different postoperative clinical manifestation of inflammatory response.

Patients and Methods In this study, 42 patients undergoing open heart surgery with the use of cardiopulmonary bypass were included and divided in two groups according to the extent of clinical manifestation of inflammatory response: Group A ( $n=21)$ patients with different severity of systemic inflammatory response syndrome (SIRS) and Group B ( $n=21)$-patients with uneventful postoperative period (no SIRS). The serum levels of PTX3 and CRP were evaluated and compared at the following time points: before and at the end of surgery, 6 hours, 1st, 3rd, and 7th day after surgery.

Results The dynamics of CRP levels were comparable between both groups and showed the classical characteristics after cardiac surgery with a peak on the 3rd postoperative day (113 vs. $132 \mathrm{mg} / \mathrm{L}$ ). In contrast, the dynamics of PTX3 showed an earlier increase of serum levels with the peak on the 1 st postoperative day in both groups ( 36.3 vs. $42.7 \mathrm{ng} / \mathrm{mL}$ ). Importantly, a significant difference of PTX3 levels was found on the $3 r d$ postoperative day $(31.1 \mathrm{vs} .7 .0 \mathrm{ng} / \mathrm{mL} ; p<0.006)$ between the two groups showing significantly delayed decrease of PTX3 levels in patients with SIRS (Group A). Conclusion This study demonstrates considerably different dynamics of PTX3 levels after cardiac surgery in patients with SIRS and patients without SIRS, thus it may be indicative to start the appropriate therapy.
\end{abstract}

\section{Introduction}

Cardiac surgery is well established for development of systemic inflammatory response. ${ }^{1,2}$ The crucial point is to achieve the physiological level of the inflammatory response

received

April 19, 2014

accepted after revision

May 23, 2014

published online

August 22, 2014 during postoperative period. In majority of patients, this response has no clinical manifestation. In minority of patients, it can result in a systemic inflammatory response syndrome (SIRS) with the risk of organ failure or even death. ${ }^{3-5}$ This undesirable reaction is based on the individual (c) 2014 Georg Thieme Verlag KG Stuttgart · New York
DOI http://dx.doi.org/ 10.1055/s-0034-1384668. ISSN 0171-6425. 
genetic predisposition together with the exposition of patients to the danger stimuli, both endogenous and exogenous origin. Endogenous danger stimuli are induced especially by oxidative stress associated with ischemia/reperfusion injury. ${ }^{6}$ Patients are heavily exposed to microbial danger patterns, which are penetrating into the body due to mucosal barrier failure. Danger signals are identified by the pattern recognition molecules (PRMs), ${ }^{7}$ which are classified into two major groups: (1) cell-associated receptors and (2) fluid-phase molecules. Fluid-phase PRMs are essential effectors and modulators of the innate immunity including collectins, ficolins, and pentraxins. ${ }^{8,9}$ Pentraxins are pivotal components of the innate immune system and important acute phase reactants. On the basis of the primary structure, pentraxins are divided into two groups: short and long pentraxins. ${ }^{10}$ C-reactive protein (CRP) is prototypic short pentraxin, whereas pentraxin 3 (PTX3) represents the long pentraxin arm of the family. ${ }^{11}$ CRP is synthesized in the liver and is barely detectable in the plasma of healthy human adults ( $\leq 3 \mathrm{mg} / \mathrm{L}$ ). Its concentration increases by as much as 1,000 -fold in several pathological conditions with maximum at approximately 48 hours. ${ }^{12}$ PTX3 is synthesized locally at the inflammatory site by both somatic and immune cells. PTX3 plasma levels are very low in normal conditions $(\leq 2 \mathrm{ng} / \mathrm{mL})^{13}$ increasing rapidly in several pathological conditions and reaching peak values after 6 to 8 hours. $^{14}$

Based on the current knowledge, it seems there is only slight increase of the PTX3 in patients during and after uncomplicated coronary artery bypass grafting (CABG) surgery with no signs of SIRS. The inherent pro-inflammatory/ anti-inflammatory response is able to deal with this clinical situation without relevant deviations of plasma PTX3 levels. In occurrence of eventful postoperative course with clinical manifestation of excessive inflammatory response it is still unknown, if the timely increase of PTX3 plasma levels can anticipate an oncoming (multiple) organ failure, so that proper treatment may be instituted as early as possible. ${ }^{6}$

The aim of this study was to compare the dynamics of two inflammatory response biomarkers (PTX3 and CRP) in two groups of patients with different clinical manifestation of inflammatory response after cardiosurgical procedure (patients with different severity of SIRS and/or its complication vs. patients with uneventful postoperative course-no SIRS) to identify cardiosurgical patients at the risk of development of excessive inflammatory response.

\section{Patients and Methods}

\section{Study Population and Clinical Characteristics}

The institutional review board approved the study and an informed consent was obtained from each patient.

A series of 42 patients undergoing open heart surgery with the use of cardiopulmonary bypass (CPB) at our institution between May 2010 and July 2012 was included in the study. Patients were divided into the following groups according to the extent of clinical manifestation of inflammatory response: Group A $(n=21)$-patients with different severity of SIRS and/or its complications and Group B $(n=21)-$ patients with uneventful postoperative period (no SIRS). SIRS was defined by at least two of following criteria: core temperature $>38^{\circ} \mathrm{C}$ or $<36^{\circ} \mathrm{C}$; heart rate $>90$ beats $/ \mathrm{min}$; respiratory rate $>20$ breaths $/ \mathrm{min}$ or $\mathrm{PaCO}_{2}<32 \mathrm{~mm} \mathrm{Hg}$; white blood cells $>12,000 / \mathrm{mm}^{3},<4,000 / \mathrm{mm}^{3}$, or $>10 \%$ immature (band) forms and always presence of clinical signs of hypoperfusion and/or organ dysfunction ${ }^{15}$ with the need for multiple vasoactive therapy and glucocorticoids (GCs) administration to maintain a mean blood pressure $>70 \mathrm{~mm}$ Hg. Patients with preoperative GCs and nonsteroid antiinflammatory drugs therapy and patients with preoperatively diagnosed infection were excluded from the study.

A prospective collection of patients' clinical data was performed. Demographic data and preoperative clinical characteristics, according to the postoperative course, are summarized in - Table 1.

\section{Anesthesiological Management}

All patients were anesthetized according to the standardized protocol. Food and fluid intake was discontinued at midnight on the day preceding surgery. Anesthesia was induced using thiopental, propofol, midazolam, or etomidate, where appropriate. Muscle relaxation was achieved with rocuronium. Anesthesia was maintained with isoflurane and intermittent administration of sufentanil. Volume-controlled ventilation with $\mathrm{FiO}_{2} 0.5$ was employed. Mean arterial pressure was kept above $70 \mathrm{~mm} \mathrm{Hg}$ by norepinephrine administered as required.

\section{Surgical Technique}

After median sternotomy and pericardiotomy, a heparin was administered intravenously ( $4 \mathrm{mg} / \mathrm{kg}$ of body weight) to maintain an activated clotting time above 480 seconds. CPB was then established by ascending aorta or subclavian artery cannulation (depending on the distal extension of the aortic disease) and by two-stage venous cannulation of the right atrium or direct bicaval venous cannulation, where appropriate. After initiation of $\mathrm{CPB}$, the pump flow was maintained at targeted rate of $2.4 \mathrm{~L} / \mathrm{min} / \mathrm{m}^{2}$. After crossclamping the aorta, cardiac arrest was achieved by administration of cold blood cardioplegic solution (St. Thomas solution, Ardeapharma, Sevetin, Czech Republic; ratio 4:1) in antegrade fashion (10-15 mL/kg of body weight). The heart was cooled topically and cardioplegia repeated every 25 minutes. The patient's temperature was allowed to drift to 34 to $35^{\circ} \mathrm{C}$. Mean arterial pressure during CPB was maintained at 50 to $70 \mathrm{~mm} \mathrm{Hg}$, hematocrit was above 0.20 . The acid-base status was kept using the $\alpha$-stat perfusion strategy. After termination of $\mathrm{CPB}$, heparin was neutralized with protamine sulfate at $1: 1$ ratio.

The CPB circuit consisted of noncoated tubing, a hard shell venous reservoir (Dideco SrL, Mirandola, Italy), a cardiotomy suction device, a hollow fiber membrane oxygenator (Dideco SrL, Mirandola, Italy), a roller pump with nonpulsatile flow (Stöckert S3, Sorin Group, München, Germany), and an arterial line filter (Dideco SrL). The extracorporeal circuit was primed with $500 \mathrm{~mL}$ of Ringer's lactate, $500 \mathrm{~mL}$ of rheodextran, 5,000 IU heparin, $80 \mathrm{~mL}$ of natrium bicarbonate $\left(\mathrm{NaHCO}_{3} 8.4 \%\right), 20 \mathrm{~mL}$ of $10 \%$ magnesium sulfate, and 
Table 1 The preoperative data

\begin{tabular}{|c|c|c|c|}
\hline & Group A & Group B & $p$ value \\
\hline Number of patients & 21 & 21 & \\
\hline Age, median (range), y & $68(38-82)$ & $62(27-76)$ & 0.232 \\
\hline Female gender & $6(28.6 \%)$ & $9(42.9 \%)$ & 0.334 \\
\hline Mean $\mathrm{BMI} \pm \mathrm{SD}$ & $28 \pm 5$ & $29 \pm 4$ & 0.848 \\
\hline Hypertension & $16(76.2 \%)$ & $16(76.2 \%)$ & 1.000 \\
\hline Dyslipidemia & $12(57.1 \%)$ & $11(52.4 \%)$ & 0.757 \\
\hline DM & $6(28.6 \%)$ & $3(14.3 \%)$ & 1.000 \\
\hline COPD & $5(23.8 \%)$ & 0 & 0.048 \\
\hline Atrial fibrillation & 9 (42.9\%) & $1(4.8 \%)$ & 0.009 \\
\hline Previous cardiac surgery & $3(14.3 \%)$ & $2(9.5 \%)$ & 1.000 \\
\hline Logistic EuroSCORE I, median (range), \% & $9.6(1.5-37.9)$ & $4.7(0.9-16.8)$ & 0.097 \\
\hline \multicolumn{4}{|l|}{ LV ejection fraction, \% } \\
\hline$>50$ & $10(47.6 \%)$ & $17(81.0 \%)$ & \multirow{3}{*}{0.057} \\
\hline $30-50$ & $9(42.9 \%)$ & $4(19.0 \%)$ & \\
\hline$<30$ & $2(9.5 \%)$ & 0 & \\
\hline
\end{tabular}

Abbreviations: BMI, body mass index; COPD, chronic obstructive pulmonary disease; DM, diabetes mellitus; LV, left ventricular; SD, standard deviation. Note: Group A-patients with different severity of SIRS and/or its complications; Group B-patients with uneventful postoperative period.

mannitol (at $1 \mathrm{~g} / \mathrm{kg}$ of body weight) and calculated to reach hematocrit level above 0.22 .

It was a heterogenous group of operations, including alone CABG, valve surgery, aortic surgery, or combination of all of them.

\section{Sample Collection and Data Acquisition}

Blood samples were withdrawn from the subclavian or antebrachial vein into both BD Vacutainer EDTA and BD Vacutainer (BD, Plymouth, UK) with a thrombin additive tubes at the following time points: before surgery, at the end of surgery, 6 hours, $1 \mathrm{st}, 3 \mathrm{rd}$, and 7 th days after surgery. Plasma and serum were separated by centrifugation and stored at $-70^{\circ} \mathrm{C}$. Plasma levels of PTX3 were determined with antihuman PTX3 ELISA kit (R\&D Systems, Minneapolis, Minnesota, United States). Assay range was 0.15 to $200 \mathrm{ng} / \mathrm{mL}$. Values above the standard curve range were extrapolated. CRP level in serum was measured using immunonephelometry. CRP was analyzed on Immage 800 (Beckman Coulter, Inc., Brea, California, United States) with Beckman Coulter CRP nephelometry kit. Detected range of values was 1 to $960 \mathrm{mg} / \mathrm{L}$.

\section{Early Postoperative Management}

Immediately after operation, all patients were transferred to the cardiosurgical intensive care unit (ICU). They were fully anesthetized, required full mechanical ventilation, and treated for hypothermia. An adequate management of potential complications was maintained. Due to the hemodynamic status, the different fluid and vasoactive drug therapy was necessary. The patients in Group A were treated with norepinephrine and at least one of inotropic drugs (dobutamine, milrinone, levosimendan). On the basis of the clinical mani- festation of SIRS and/or its complications (signs of hypoperfusion and/or organ dysfunction), a glucocorticosteroids therapy was initiated from the operation day to the 3rd postoperative day. The type of GCs, dose, and duration of therapy was defined at discretion of an independent physician in charge. The dose ranged from 200 to $5,200 \mathrm{mg}$ of hydrocortisone equivalent (median $700 \mathrm{mg}$ ) administrated continuously or intermittent. The patients in Group B were treated with no vasoactive drugs or GCs.

\section{Statistical Analysis}

Continuous and discrete variables were reported as mean \pm standard deviation or median and range for data not normally distributed. Categorical and ordinal variables were reported using the number and percentage of observations. Continuous and discrete variables were compared using a twosample $t$-test, Mann-Whitney or Kolmogorov-Smirnov test, where appropriate. Categorical and ordinal variables were compared using a Pearson chi-squared test or Fischer exact test, where appropriate. A $p$ value $<0.05$ was considered to indicate statistical significance. Statistical analysis was performed using the IBM SPSS Statistics software program (version 21.0.0.0 for MS Windows, IBM Corporation, Armonk, New York, United States) and Statistica 10 software (StatSoft, Inc., Tulsa, Oklahoma, United States).

\section{Results}

\section{Surgical Data and Early Clinical Characteristics}

The operative data according to the postoperative course are shown in - Table 2. Briefly, a mitral and tricuspid valve surgery was performed in 67 and 53\% (Group A) versus 24 
Table 2 The operative and early postoperative data

\begin{tabular}{|l|l|l|l|}
\hline & Group A & Group B & $p$ value \\
\hline Number of patients & 21 & 21 & \\
\hline CABG & $11(52.4 \%)$ & $8(38.1 \%)$ & 0.352 \\
\hline Aortic valve surgery & $10(47.6 \%)$ & $15(71.4 \%)$ & 0.116 \\
\hline Mitral valve surgery & $14(66.7 \%)$ & $5(23.8 \%)$ & 0.005 \\
\hline Tricuspid valve surgery & $11(52.4 \%)$ & $3(14.3 \%)$ & 0.020 \\
\hline Surgery on the aorta & $3(14.3 \%)$ & $6(28.6 \%)$ & 0.454 \\
\hline CPB time, median (range), min & $130(72-301)$ & $110(54-208)$ & 0.450 \\
\hline Cross-clamp time, median (range), min & $94(43-242)$ & $87(37-180)$ & 0.443 \\
\hline Chest tube output 24 h, median (range), mL & $800(300-3,050)$ & $400(150-2,000)$ & 0.007 \\
\hline Re-exploration for bleeding or tamponade & $2(9.5 \%)$ & $1(4.8 \%)$ & 1.000 \\
\hline Ventilation time, median (range), h & $26(5-265)$ & $7(3-20)$ & $<0.001$ \\
\hline Reintubation & $4(19.0 \%)$ & 0 & 0.107 \\
\hline ARDS & $6(28.6 \%)$ & 0 & 0.021 \\
\hline Acute renal failure requiring dialysis & $4(19.0 \%)$ & 0 & 0.107 \\
\hline Stroke & $2(9.5 \%)$ & 0 & 0.488 \\
\hline Multiorgan failure & $2(9.5 \%)$ & 0 & 0.488 \\
\hline Atrial fibrillation & $16(76.2 \%)$ & $4(19.0 \%)$ & 0.001 \\
\hline DSWI & 0 & 0 & \\
\hline ICU length of stay, median (range), h & $119(21-996)$ & $45(15-96)$ & $<0.001$ \\
\hline Hospital length of stay, median (range), $\mathrm{d}$ & $20(12-90)$ & $10(8-53)$ & $<0.001$ \\
\hline 30-day mortality & 0 & 0 & \\
\hline
\end{tabular}

Abbreviations: ARDS, acute respiratory distress syndrome; CABG, coronary artery bypasses grafting; CPB, cardiopulmonary bypass; DSWI, deep sternal wound infection; ICU, intensive care unit.

Note: Group A-patients with different severity of SIRS and/or its complications; Group B-patients with uneventful postoperative period.

and $14 \%$ (Group B), respectively $(p=0.005$ and $p=0.02$ ). The CPB time/aortic cross-clamp time was $130 / 94$ minutes in Group A versus 110/87 minutes in Group B, demonstrating no statistically significant difference between the groups ( $p=0.450$ and $p=0.443$ ). Blood loss was $800 \mathrm{~mL} / 24$ hours in Group A versus $400 \mathrm{~mL} / 24$ hours in Group B $(p=0.007)$. There were significant differences between Group A versus Group B in ventilation time 26 versus 7 hours, ICU length of stay 119 versus 45 hours, and hospital length of stay 20 versus 10 days, respectively ( $p<0.001$ in all). Need of reintubation, occurrence of acute respiratory distress syndrome, acute renal failure requiring dialysis, stroke, or atrial fibrillation were more frequent in Group A, but not reaching a statistical significance. No death, acute myocardial infarction (AMI), or deep sternal wound infection occurred.

\section{Samples Analysis-Pentraxin 3 and C-Reactive Protein}

\section{Serum Levels of PTX3}

The preoperative serum level of PTX3 was $0.6 \mathrm{ng} / \mathrm{mL}$ in Group A and $0.4 \mathrm{ng} / \mathrm{mL}$ in Group B, respectively. Increased serum levels were observed after termination of surgery and the highest PTX3 level throughout the whole observation was measured on the 1st postoperative day (36.3 vs. $42.7 \mathrm{ng} / \mathrm{mL}$; $p>0.60$ ). Afterward, the PTX3 levels were decreasing. On the 3rd postoperative day, the PTX3 levels remained substantially elevated in Group A (31.1 ng/mL) than in Group B $(7.0 \mathrm{ng} / \mathrm{mL})$. This was the only statistically significant difference in dynamics of PTX3 levels observed between the two groups $(p<0.006)$. Elevated serum levels of PTX3 remained as long as to the 7th postoperative day ( $4.8 \mathrm{vs.} 2.7 \mathrm{ng} / \mathrm{mL} ; p<0.15$ ) (-Fig. 1).

\section{Serum Levels of C-Reactive Protein}

A similar dynamics of the CRP levels was observed in both groups, but did not significantly differ between the two groups during the whole observation period. Baseline levels of serum CRP were $0.6 \mathrm{mg} / \mathrm{L}$ in Group A and $0.4 \mathrm{mg} / \mathrm{L}$ in Group B. They increased 6 hours after surgery, reaching the peak of 113 versus $132 \mathrm{mg} / \mathrm{L}$ on the 3rd postoperative day $(p=0.615)$. The elevated CRP serum levels remained as long as to the 7th postoperative day $(26.0 \mathrm{mg} / \mathrm{L}$ in Group A vs. $26.2 \mathrm{mg} / \mathrm{L}$ in Group B; $p=0.73$ ) (-Fig. 2).

\section{Comparison of Pentraxin 3 and C-Reactive Protein Serum Levels}

The increase of PTX3 level started immediately after the surgery termination and reached the maximum on the 1 st 


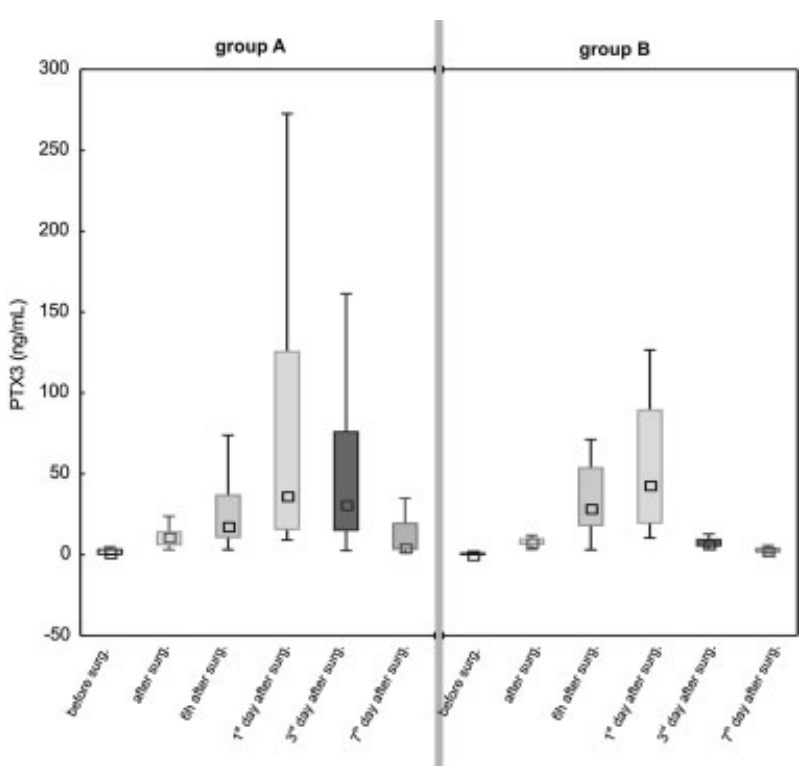

Fig. 1 PTX3 levels in serum of patients undergoing cardiac surgery (Group A-patients with different severity of SIRS and/or its complications; Group B-patients with uneventful postoperative period). The level of PTX3 differed significantly on the 3rd postoperative day between the two groups $(p<0.006)$. The PTX3 levels remained more elevated in Group A up to the 7th postoperative day $(p<0.15)$. Squares display median, boxes are quartiles, and whiskers display the range of nonoutlier values. PTX3, pentraxin 3; SIRS, systemic inflammatory response syndrome.

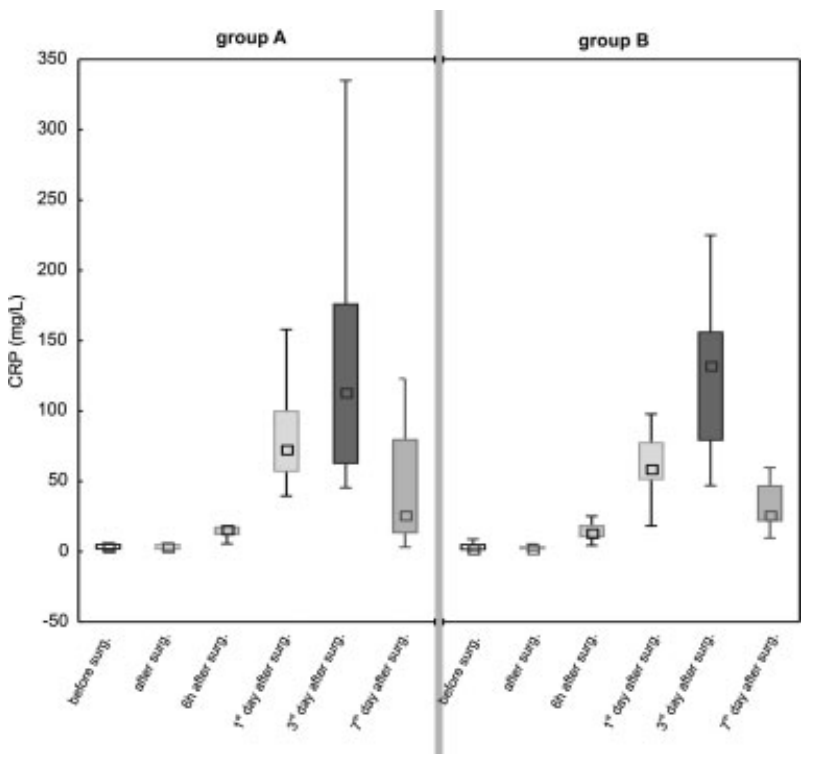

Fig. 2 CRP levels in serum of patients undergoing cardiac surgery (Group A-patients with different severity of SIRS and/or its complications; Group B-patients with uneventful postoperative period). The level of CRP did not differ significantly at all time points between the two groups. Squares display median, boxes are quartiles, and whiskers display the range of nonoutlier values. CRP, C-reactive protein; SIRS, systemic inflammatory response syndrome.

postoperative day in both groups. The release of CRP was delayed in comparison to PTX3 with the increase beginning 6 hours after surgery and peaking on the 3rd postoperative day in both groups. The serum levels of both biomarkers remained elevated as long as to the 7th postoperative day; however, the

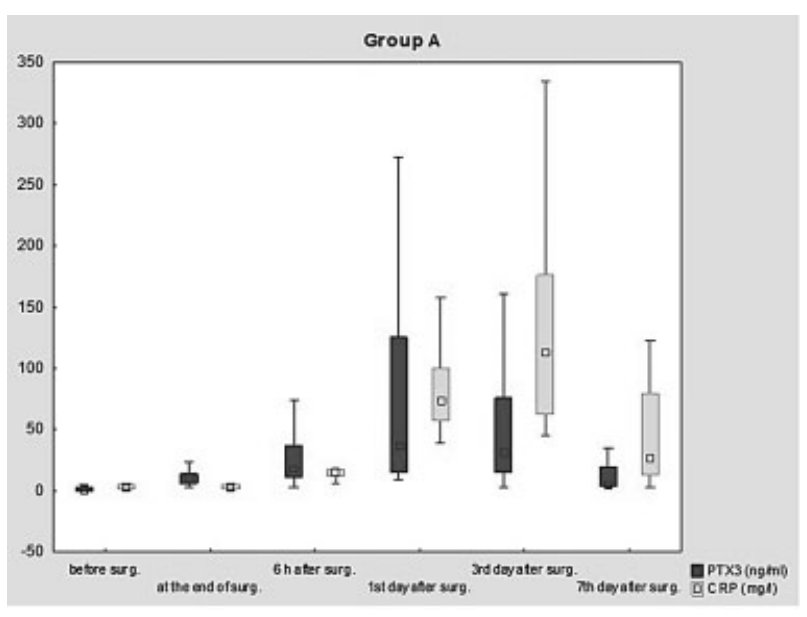

Fig. 3 PTX3 and CRP levels in serum of patients undergoing cardiac surgery within Group A-patients with different severity of SIRS and/or its complications. Plots show the early increase of PTX 3 level and the delayed release of CRP. Both biomarkers remained elevated as long as to the 7th postoperative day; however, the decrease of CRP level was slow compared with the PTX level. Squares display median, boxes are quartiles, and whiskers display the range of nonoutlier values. CRP, Creactive protein; PTX3, pentraxin 3; SIRS, systemic inflammatory response syndrome.

decrease of CRP levels was proportionally slow in both groups and the PTX3 level decrease even more significant in Group B (-Figs. 3 and 4 ).

\section{Discussion}

Cardiosurgical operation, especially with the use of CPB, served as a model of inflammatory response characterized by increased level of both proinflammatory and anti-

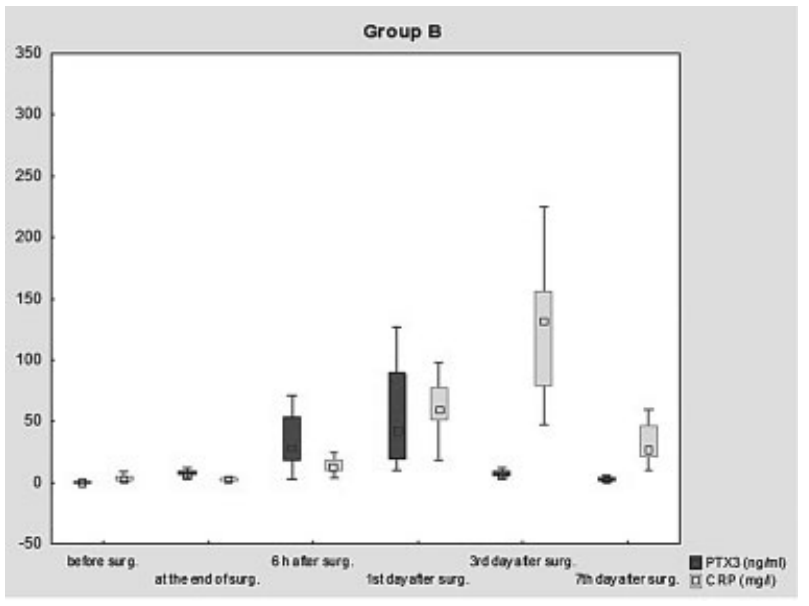

Fig. 4 PTX3 and CRP levels in serum of patients undergoing cardiac surgery within Group B-patients with uneventful postoperative period. Plots show the early increase of PTX3 level and the delayed release of CRP. Both biomarkers remained elevated as long as to the 7th postoperative day; however, the decrease of CRP level was slow compared with the PTX3 level (PTX3 level decrease even more significant in this group). Squares display median, boxes are quartiles, and whiskers display the range of nonoutlier values. CRP, C-reactive protein; PTX3, pentraxin 3. 
inflammatory mediators. ${ }^{1,2}$ An excessive inflammatory response can have clinical manifestation and lead to postoperative complications, organ dysfunction, or failure. An attempt is to recognize this unwanted reaction as soon as possible and to initiate the treatment to reduce the postoperative morbidity and mortality.

The current study is one of few to evaluate and compare the serum levels of PTX3 and CRP in patients undergoing cardiac surgery with different postoperative course. The CRP serum levels in our study were similar in both groups and do not correlate with the extent of excessive inflammatory response. On the contrary, we found statistically significant differences in PTX3 levels on the 3rd postoperative day between the two groups. The PTX3 levels remained more elevated up to the 7th postoperative day in the group of patients with eventful postoperative period (Group A), defined by the postoperative signs of hypoperfusion and/or organ failure (clinically manifested SIRS and/or its complications) with administration of multiple vasoactive agents and GCs therapy. However, we expected significant difference of the PTX3 serum levels between the groups already on the 1st postoperative day, this fact was not confirmed. The reason could be that cardiosurgical operations have generally more prolonged and complex postoperative course and therefore the potential complications due to excessive inflammatory response may occur later.

The dynamics of both biomarkers evaluated in our study were similar to that published by Kunes et al in a study comparing patients undergoing uncomplicated CABG with or without the use of CPB in 2007. ${ }^{16}$ They described the peak of CRP levels on the 3rd postoperative day and the peak of PTX3 levels on the 1st postoperative day, respectively. Their patients have postoperatively no signs of excessive inflammatory response and the serum levels of PTX3 were not increased above the upper limit of normal at all. Another study showing similar dynamics of PTX3 in murine models of acute myocardial infarction (AMI) was published by Salio et al. ${ }^{17}$ They found the PTX3 serum levels increase starting 8 hours after permanent coronary occlusion, peaked at 24 hours, and remained elevated up to 72 hours. Unlike, in a study published by Peri et al of 37 patients with AMI, the PTX3 peaked much earlier (median 7.5 hours) than CRP (median 24 hours) after AMI $(p=0.001){ }^{18}$ The reason of the delayed PTX3 peak in our study may be caused by longer period between the two blood sampling points ( 6 hours after termination of surgery and the 1 st postoperative day). In this timeframe, the real peak may have been hidden. On the contrary, in the study of Peri et al, ${ }^{18}$ the first blood sampling point was defined at the coronary care unit admission, which is not always the time point of symptoms onset of an AMI. Taking this into consideration, the real peak may have been some hours later.

This study also showed significantly higher PTX3 serum levels than into the date published studies in adult cardiovascular medicine, ${ }^{16,18-20}$ whereas the CRP levels are comparable to that published in cardiosurgical studies. ${ }^{5,16,21}$ Based on our results, it seems the higher PTX3 levels may correlate to the complexity of the cardiac operation. This postulation could be supported by results of Kunes et al, where only CABG patients ("on-pump" vs. "off-pump") with uneventful postoperative course were included. ${ }^{16}$

Doni et al indicated in their study that the regulation of PTX3 by GCs is dependent on the cell type, with inhibition in hematopoietic cells (macrophages and dendritic cells) and induction in nonhematopoietic cells (fibroblasts and endothelial cells). They concluded in vivo administration of GCs might increase the blood levels of PTX3 in mice and humans. ${ }^{22}$ On the contrary, results published by Kunes et al do not correspond with this fact. ${ }^{16}$ They found no significant PTX3 increase (above normal level of $2 \mathrm{ng} / \mathrm{mL}$ ) in patients undergoing $\mathrm{CABG}$ with uneventful postoperative course, despite the patients in the $\mathrm{CPB}$ group were administered $500 \mathrm{mg}$ methylprednisolone into the CPB priming solution (equivalent to $2,500 \mathrm{mg}$ of hydrocortisone).

\section{Study Limitations}

This study has several limitations being primarily the definition and the clinical heterogeneity of SIRS. Obviously, we defined SIRS according to latest knowledge. However, the definition may be regarded as weak. Still, we think that this may be the most valid approximation to the problem. The PTX3 levels in Group A may be influenced by administration of GCs.

\section{Conclusion}

This study shows a significant difference in serum levels of PTX3 in patients with and without SIRS after open heart surgery. Consequently, this newly introduced biomarker may serve as an extension of the armamentarium of the clinician to diagnose and treat SIRS in the postoperative period. Based on current knowledge, the cutoff level of PTX3 for developing an excessive inflammatory response with clinical manifestation remains still unclear. Further studies are needed to elucidate the real diagnostic and prognostic value of PTX3.

\section{Acknowledgments}

This work was supported by the project of Ministry of Health, Czech Republic for conceptual development of research organization (00179906) and by the program of Charles University Prague, Czech Republic (PRVOUK P37/04).

Note

This article was presented at the 32nd Cardiovascular Surgical Symposium-CSS, Zürs, Austria, March 22-29, 2014.

Conflict of Interest

None.

\section{References}

1 Partrick DA, Moore EE, Fullerton DA, Barnett CC Jr, Meldrum DR, Silliman CC. Cardiopulmonary bypass renders patients at risk for multiple organ failure via early neutrophil priming and late neutrophil disability. J Surg Res 1999;86(1):42-49 
2 Svitek V, Lonsky V, Anjum F. Pathophysiological aspects of cardiotomy suction usage. Perfusion 2010;25(3):147-152

3 Chew MS, Brandslund I, Brix-Christensen V, et al. Tissue injury and the inflammatory response to pediatric cardiac surgery with cardiopulmonary bypass: a descriptive study. Anesthesiology 2001;94(5):745-753

4 Chowdhury UK, Malik V, Yadav R, et al. Myocardial injury in coronary artery bypass grafting: on-pump versus off-pump comparison by measuring high-sensitivity C-reactive protein, cardiac troponin I, heart-type fatty acid-binding protein, creatine kinaseMB, and myoglobin release. J Thorac Cardiovasc Surg 2008;135(5): 1110-1119, e1-e10

5 Bical OM, Fromes Y, Gaillard D, et al. Comparison of the inflammatory response between miniaturized and standard CPB circuits in aortic valve surgery. Eur J Cardiothorac Surg 2006;29(5):699-702

6 Kunes P, Mandak J, Holubcova Z, Kolackova M, Krejsek J. The long pentraxin PTX3: a candidate anti-inflammatory mediator in cardiac surgery. Perfusion 2013;28(5):377-389

7 Iwasaki A, Medzhitov R. Regulation of adaptive immunity by the innate immune system. Science 2010;327(5963):291-295

8 Holmskov U, Thiel S, Jensenius JC. Collections and ficolins: humoral lectins of the innate immune defense. Annu Rev Immunol 2003; 21:547-578

9 Bottazzi B, Garlanda C, Salvatori G, Jeannin P, Manfredi A, Mantovani A. Pentraxins as a key component of innate immunity. Curr Opin Immunol 2006;18(1):10-15

10 Deban L, Jaillon S, Garlanda C, Bottazzi B, Mantovani A. Pentraxins in innate immunity: lessons from PTX3. Cell Tissue Res 2011; 343(1):237-249

11 Garlanda C, Bottazzi B, Bastone A, Mantovani A. Pentraxins at the crossroads between innate immunity, inflammation, matrix deposition, and female fertility. Annu Rev Immunol 2005;23:337-366

12 Steel DM, Whitehead AS. The major acute phase reactants: Creactive protein, serum amyloid $P$ component and serum amyloid A protein. Immunol Today 1994;15(2):81-88
13 Yamasaki K, Kurimura M, Kasai T, Sagara M, Kodama T, Inoue K Determination of physiological plasma pentraxin 3 (PTX3) levels in healthy populations. Clin Chem Lab Med 2009;47(4): 471-477

14 Muller B, Peri G, Doni A, et al. Circulating levels of the long pentraxin PTX3 correlate with severity of infection in critically ill patients. Crit Care Med 2001;29(7):1404-1407

15 Balk RA. Systemic inflammatory response syndrome (SIRS): where did it come from and is it still relevant today? Virulence 2014;5(1): 20-26

16 Kunes P, Lonsky V, Mandak J, et al. The long pentraxin 3 in cardiac surgery: distinct responses in "on-pump" and "off-pump" patients. Scand Cardiovasc J 2007;41(3):171-179

17 Salio M, Chimenti S, De Angelis N, et al. Cardioprotective function of the long pentraxin PTX3 in acute myocardial infarction. Circulation 2008;117(8):1055-1064

18 Peri G, Introna $\mathrm{M}$, Corradi D, et al. PTX3, A prototypical long pentraxin, is an early indicator of acute myocardial infarction in humans. Circulation 2000;102(6):636-641

19 Latini R, Maggioni AP, Peri G, et al; Lipid Assessment Trial Italian Network (LATIN) Investigators. Prognostic significance of the long pentraxin PTX3 in acute myocardial infarction. Circulation 2004; 110(16):2349-2354

20 Hudzik B, Szkodzinski J, Pietka-Rzycka A, et al. Plasma pentraxin 3 may be a more sensitive marker of inflammatory response than high-sensitivity C-reactive protein after bare-metal stent compared to drug-eluting stent implantation. J Interferon Cytokine Res 2013;33(5):280-284

21 Rastan AJ, Bittner HB, Gummert JF, et al. On-pump beating heart versus off-pump coronary artery bypass surgery-evidence of pump-induced myocardial injury. Eur J Cardiothorac Surg 2005; 27(6):1057-1064

22 Doni A, Mantovani G, Porta C, et al. Cell-specific regulation of PTX3 by glucocorticoid hormones in hematopoietic and nonhematopoietic cells. J Biol Chem 2008;283(44):29983-29992 